\title{
Modified Le Gall jig - treatment guidelines in a case of severely worn dentition, full mouth rehabilitation and increased vertical dimension of occlusion
}

\author{
Ivan Chakalov', Pavlina Ivanova ${ }^{2}$. \\ 1. Department of Prosthetic Dentistry, Faculty of \\ Dental Medicine, Medical University - Sofia, \\ Bulgaria;
}

2. Dentist with a Private Practice - Sofia, Bulgaria;

\begin{abstract}
Dental wear under its different forms, such as abrasion and especially erosion, becomes more and more frequent in various populations around the globe. Recent studies indicate that the problem spreads on an epidemic scale and according to some researches the overall prevalence is estimated to be more than $30 \%$ (1) for permanent teeth of children and adolescents. All these data signify that in the near future dentists will be confronted with many patients and cases of severely worn dentitions and that the need of a comprehensive, affordable and systematic treatment approach is bigger than ever.

The paper presents a case report of the restoration of a severely worn dentition of a young patient followed up to the stage of finalized composite provisionals. The treatment follows with some minor modifications the "Geneva protocol" described by Belser and Vailati (2). The innovative part in the present case consists in the utilization of the jig of Le Gall (3) with some modifications allowing the device to be used not only in order to determine mandible centric position, but also as a useful instrument when resolving several esthetic and functional dilemmas. Thus the modified Le Gall jig becomes a treatment guideline substantively reducing the guesswork and allows even a well-trained
\end{abstract}


general practitioner to deal with complex cases such as full-mouth reconstruction with a vertical dimension of occlusion (VDO) increase.

Keywords: wear, abrasion, erosion, vertical dimension of occlusion, increased VDO

\section{Background}

Dental wear with its different forms, such as abrasion, attrition or erosion, becomes more and more frequent especially among young people. Some of the latest epidemiological studies estimate the prevalence of dental erosion in permanent teeth of children and adolescent to be more than $30,4 \%$ (1). Controversies might be found in the literature regarding the etiological factors, but whatever the causes, a clear ascending tendency is visible in the studies of different populations and countries throughout the globe, meaning that in the future more and more patients with severe dentition wear will be visiting our offices (4). Until recently the approach to such cases comprised an almost systematic root canal treatment and full-crown coverage (5). However a much more conservative systematic approach has been proposed by Vailati and Belser with the so-called three-step technique (2). The aggressive root canal treatment and full crown preparations were replaced by bonded porcelain overlays and laminate veneer restorations which presented improved results in long term follow-up. Provisional restorations might be bonded as proposed by Gurel and subsequently used as a guide during the preparation - the so-called "APTtechnique" (6).

Some important questions remain unanswered, however, such as: how much to raise the vertical dimension of occlusion (VDO), what length of the restored teeth to choose and how to communicate the new position, dimensions and spatial orientation to the laboratory.

As a guideline in this case we used a modification of a jig previously described by Le Gall (3) as a tool to deprogram the mandibular closing path as well as to determine the mandibular centric position. A characteristic feature differing the Le Gall jig from other more popular ones like e.g. the Lucia jig (7) is that it has on the palate a relatively flat area perpendicular to the closing trajectory of the lower incisors where a single centered occlusal contact is expected

\section{Case Description}

A 35 years old patient was referred to our clinic with the request for a full-mouth rehabilitation at a new increased VDO. The referring colleague was not a specialist in prosthetics and considered the treatment beyond her professional capacity. The initial situation is demonstrated on figures 1-3. Impressions were taken. 


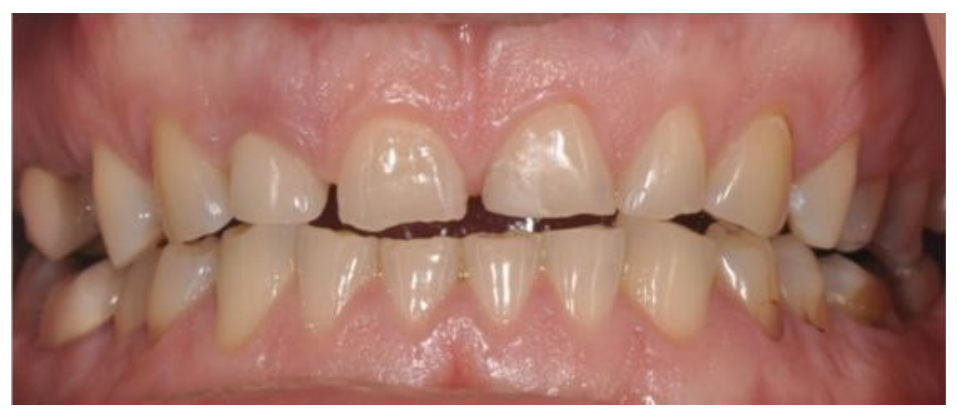

Figure 1 Initial situation

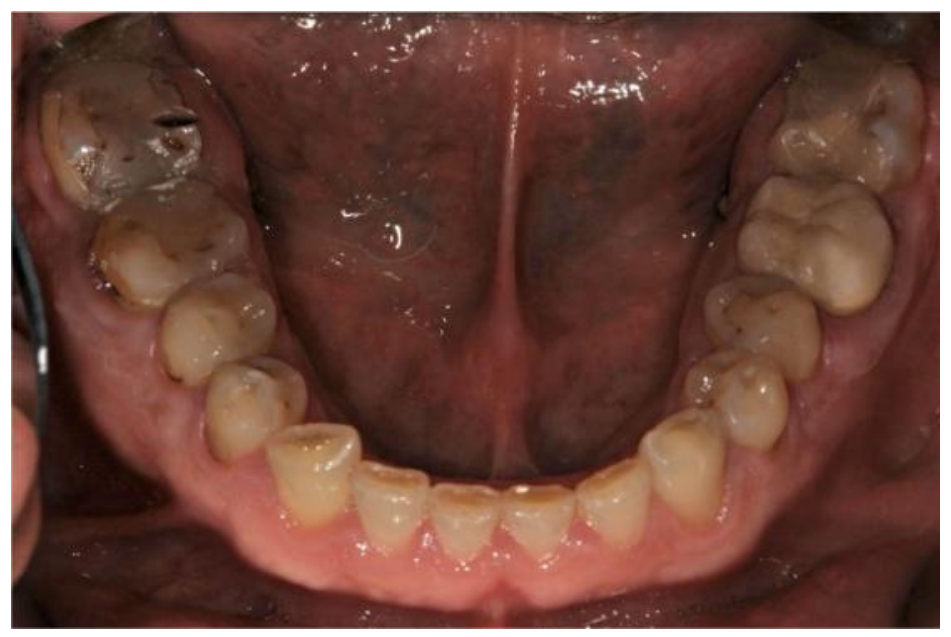

Figure 2 Initial situation 


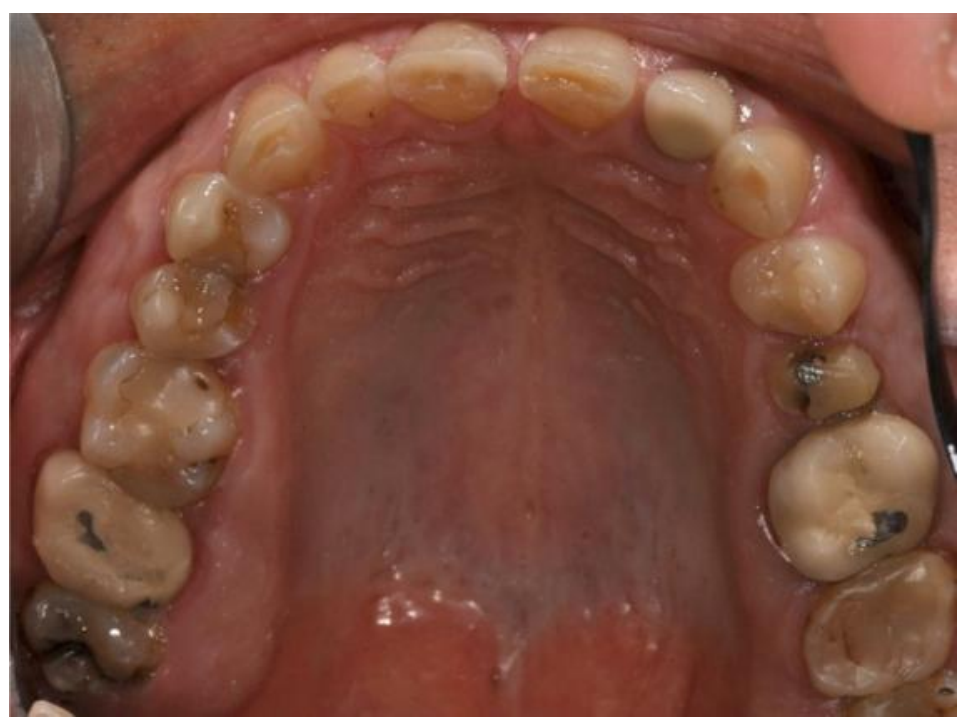

Figure 3 Initial situation

\section{Jig fabrication}

In order to mount the models in an articulator in the desired position a jig was fabricated (Fig. 4). The material of choice was Revotec (GC), but another composite or a self-curing acrylic resin might be used. On the palatal side a relatively flat area is created either by sculpting before polymerization or by grinding once the material is set. Thus a flatten area is created on the palatal side of the jig roughly perpendicular to the closing trajectory of the lower incisors. Using an articulating paper together with the jig and different tests known from total prosthetics (such as manual manipulation of the mandible, swallowing, closing with the tongue pressed against the palate) should reconfirm an identical contact on the jig (Fig. 5). Because of the paper markings even minor discrepancies in the centric position might be detected allowing the clinician to choose a position confirmed by several different methods. The jig was then modified in order to provide more information needed throughout the treatment. It was slightly elongated distally in both left and right direction in order to mimic the position of the incisal edges of the two central incisors and the plane was checked using the occlusal plane guide to obtain a horizontal reference (Fig 6). The midline was indicated in the middle of the jig to obtain a midfacial reference. The right VDO of the occlusion was then determined using the methods used for total dentures (rest position height) (Fig 7). The new VDO was then incorporated into the jig by adding material on the palatal side. The desired VDO was objectively registered by measuring the distance between the gingival zenith points of antagonistic teeth on the opposing arches. In order to validate the correct display during speech of the central incisor the patient was asked to count to twenty with the jig in the mouth. A movie was registered and the phonetics, lips movements and the tooth display were analyzed and discussed with the patient to obtain his agreement regarding the incisal border positioning (fig.8). The normal tooth display for a young male patient is considered to be about $2 \mathrm{~mm}$, but this average value might vary depending on the patient's age and sex, as well as on the preferences of both patient and practitioner (8). 


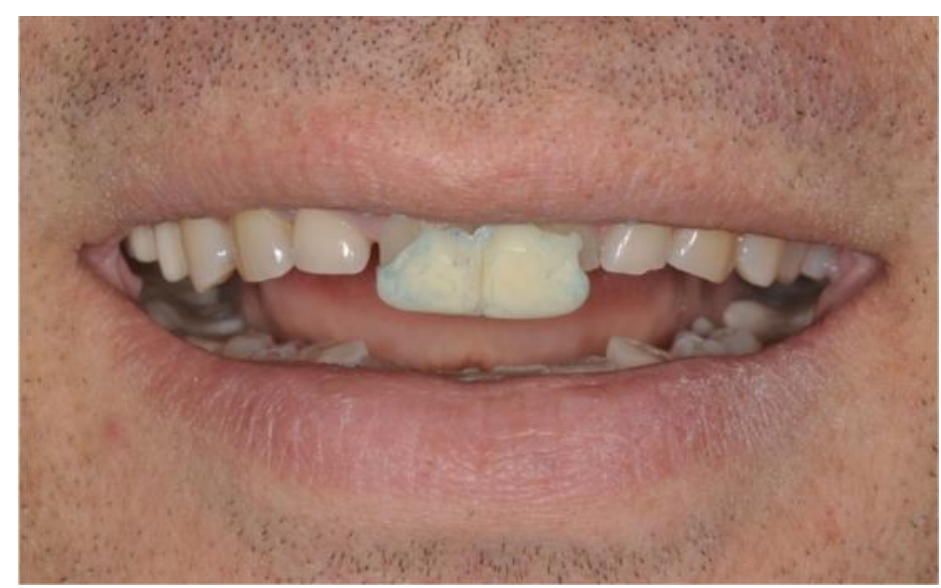

Figure 4 Jig fabrication

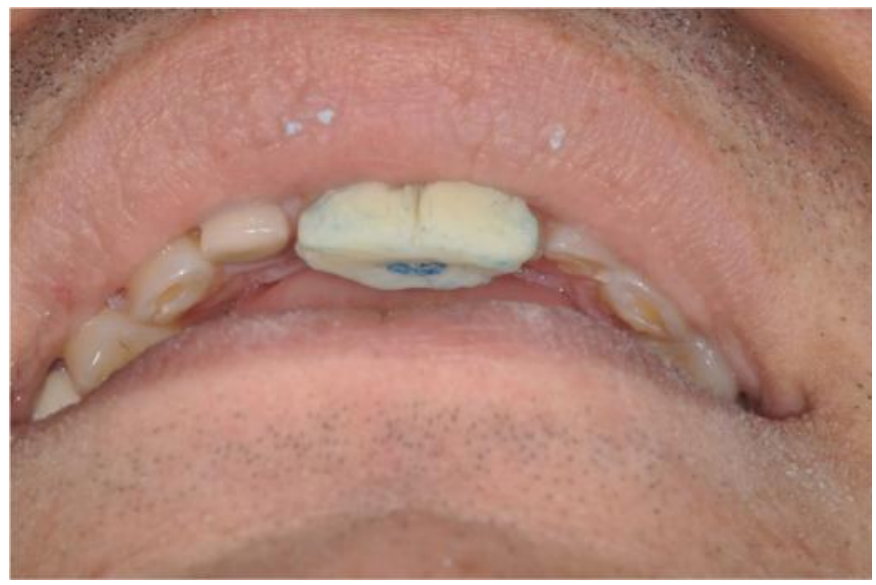

Figure 5 Symmetrical simultaneous contact with the medioincisal angle of the lower central incisors 


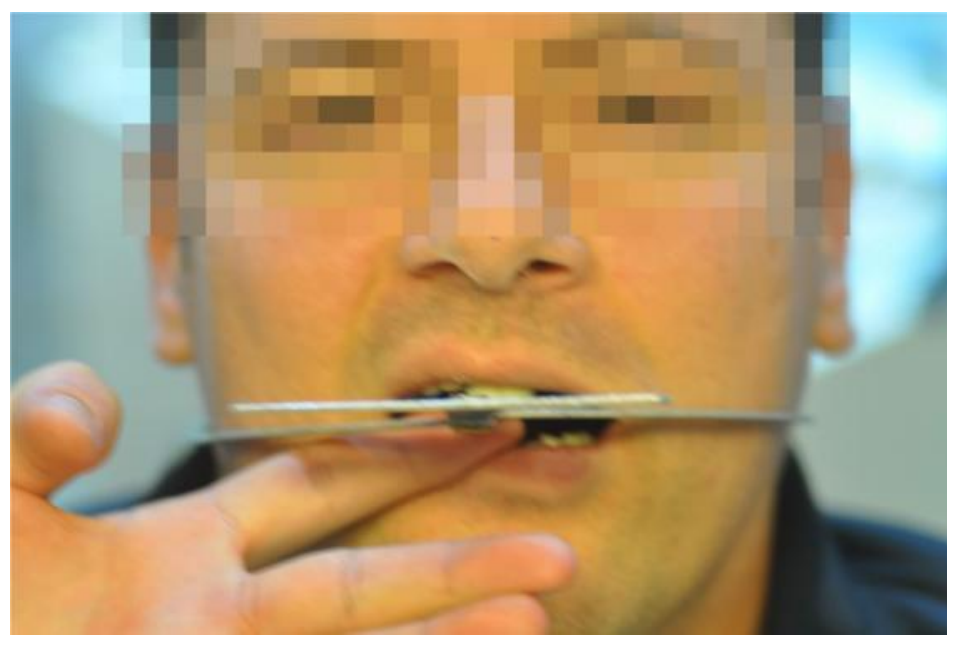

Figure 6 Checking the occlusal plane with Vinnie's gauge

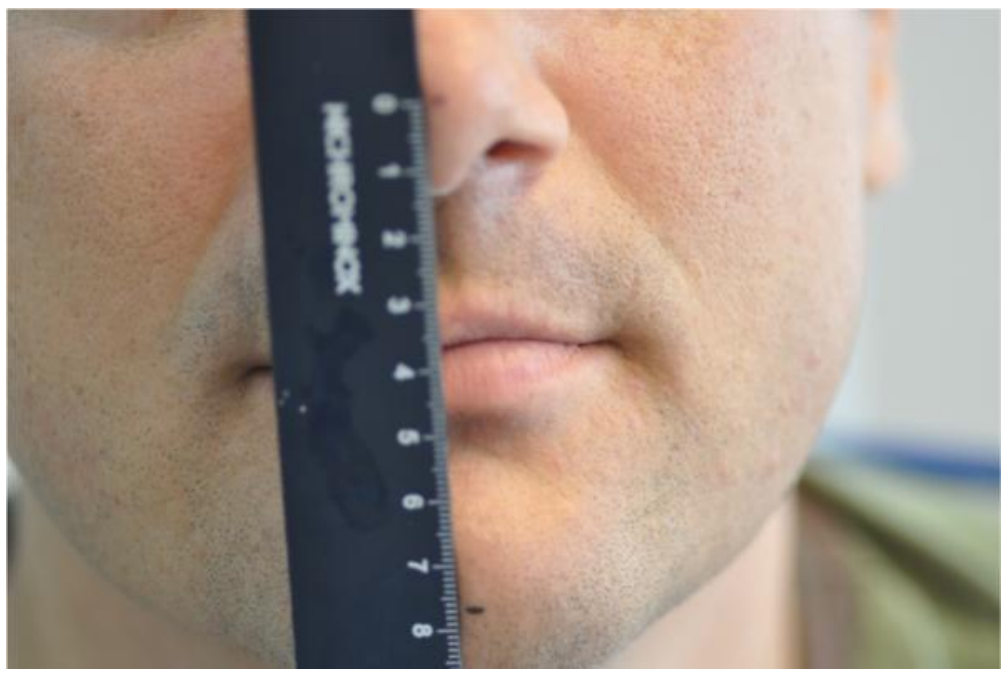

Figure 7 Rest position height 


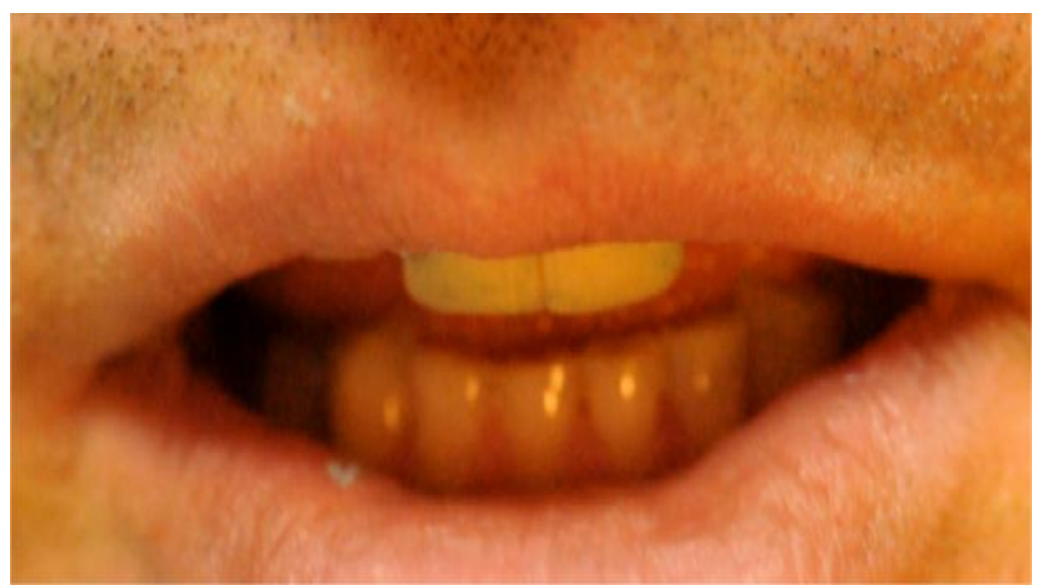

Figure 8 Incisal border positioning

\section{Bite registration}

With all these data established and validated, a bite registration might be taken. With the jig in mouth the patient was asked to close it in the centric position and the clearance between the opposing arches was filled using bite registration material (Variotime bite, Heraeus). An important sign that the registered position is the desired one is if there is a perforation of the bite material coinciding with the marked contact on the palatal side of the jig (Fig. 9).।

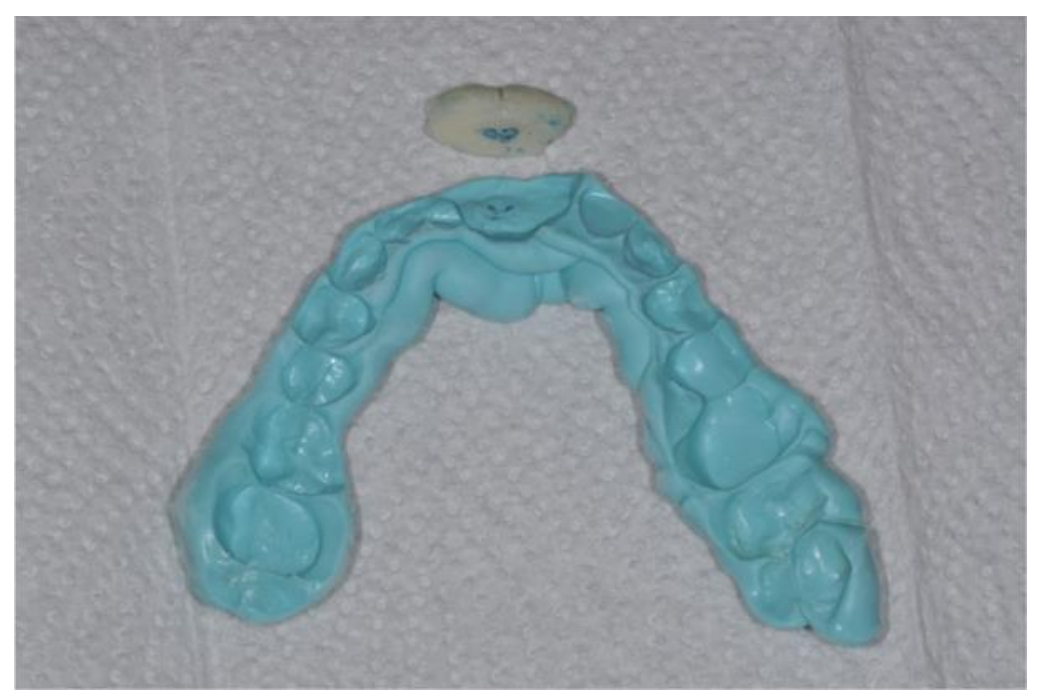

Figure 9 Bite registration

\section{Wax-up}

Models were then mounted in a semiadjustable articulator. After the mounting was complete, the distance between the zenith point of selected antagonist teeth was measured as a method to control that the clinically determined VDO was not altered. Wax-up was then fabricated (Fig.10, 11). 

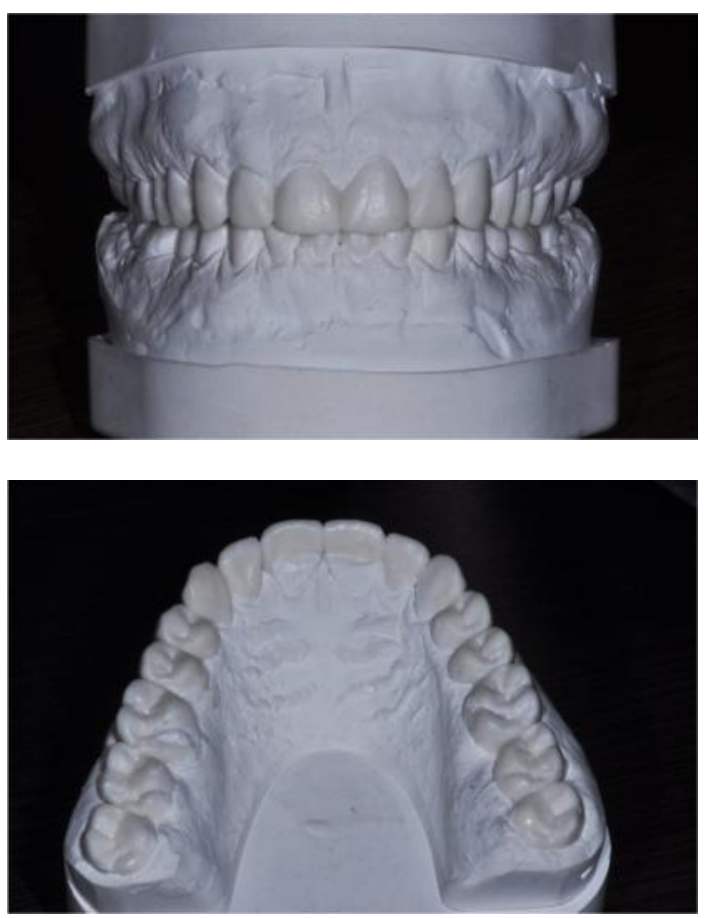

Figures 10 and 11 Wax-up

\section{Mock-up}

The shapes and size of the waxed teeth need to be validated in the mouth and approved by the patient. A transparent index made of polyvinyl siloxane impression material (Memosil, Heraeus Kulzer) was produced using the waxed models. A flowable composite (Premise Flowable, Kerr) was used for the mock-up fabrication (Fig.12, 13) and the resultant effect was analyzed and discussed with the patient (Fig.14). Special attention should be paid to the horizontality of the occlusal plane, phonetics and tooth display during moderate and exaggerated smile and during speech. It is vital to obtain the patient's approval at this stage in order to proceed with the treatment as well as to document well the situation using photos and impressions. 

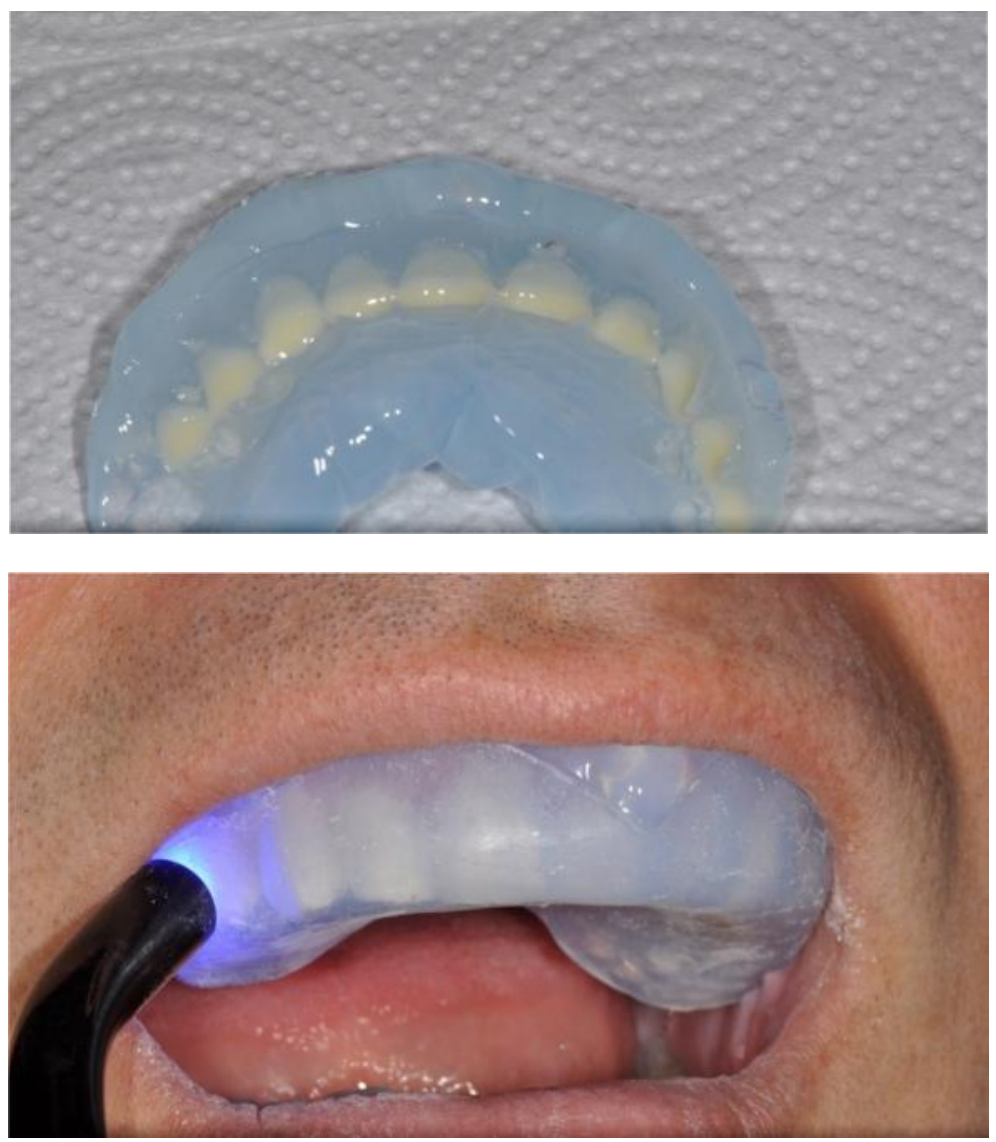

Figures 12 and 13 Mock-up fabrication

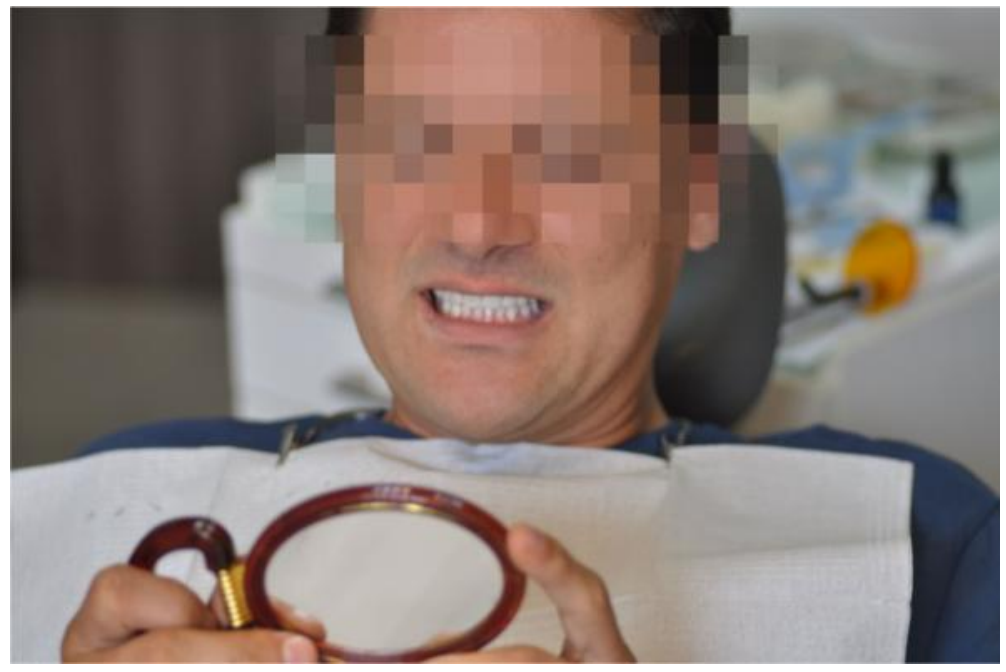

Figure 14 Discussion with the patient 


\section{Provisionals}

There are several possible methods to produce the provisionals - direct, hand-made indirect, CAD/CAM made indirect. In this case we chose the direct approach. The sequence of the teeth to be restored was chosen in such a way that the fabricated restoration could fit in the occlusion predetermined by the jig and acquire the shape and size determined by the wax-up. Transparent molds were produced based on the wax-up (two posterior and one anterior for each jaw). The teeth that were already prepared for full crowns were first restored using acrylic resin. After each pair of antagonistic crowns was fabricated the occlusion was checked and adjusted using a shimstock foil until it was blocked by both distal crowns and the jig in the front (Fig.15). The same procedure was repeated on the right side. With the occlusion now fixed between the distal crowns at the level of first molars and the jig in the front, the premolars were restored directly in the mouth using the transparent mold and composite material (Herculite HRV, Kerr). After every antagonistic couple was complete, the occlusion was systematically checked so that the shimstock was stuck between all the finished antagonists, as well as between the jig and the lower anteriors. Once occlusion was further stabilized in the premolar area, the frontal teeth were tackled (Fig. 16). After completing every restoration the tooth was cleaned from excess material and thoroughly polished. The finest polishers for ceramics under water irrigation gave the best results. The finalized provisionals are shown on Fig.17 and 18. Except for the teeth that were already prepared for full crowns $(12,17,25,26,46)$ and those that already had root canal treatment and needed full crowns $(17,38,36)$, no other teeth was prepared. With the new occlusion and the increased VDO, the new anterior crown length needed to be validated by the patient for several weeks until proceeding with the final restoration.

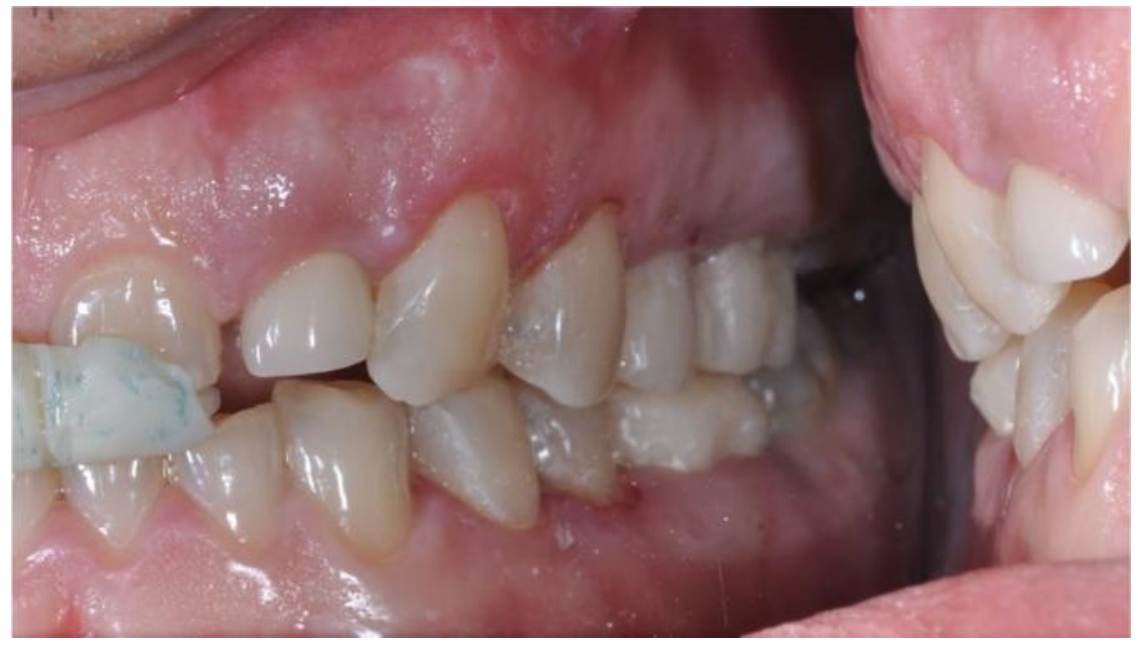

Figure 15 Contact points between the distal restorations and the jig in the front 


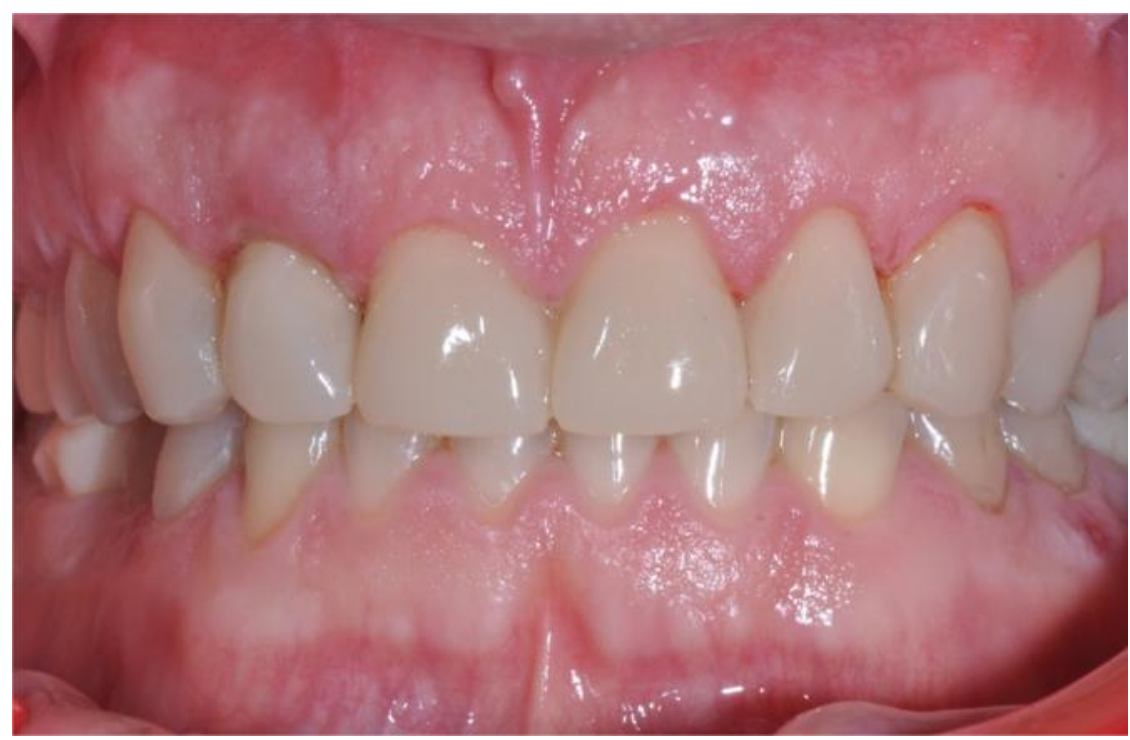

Figure 16 The final outlook
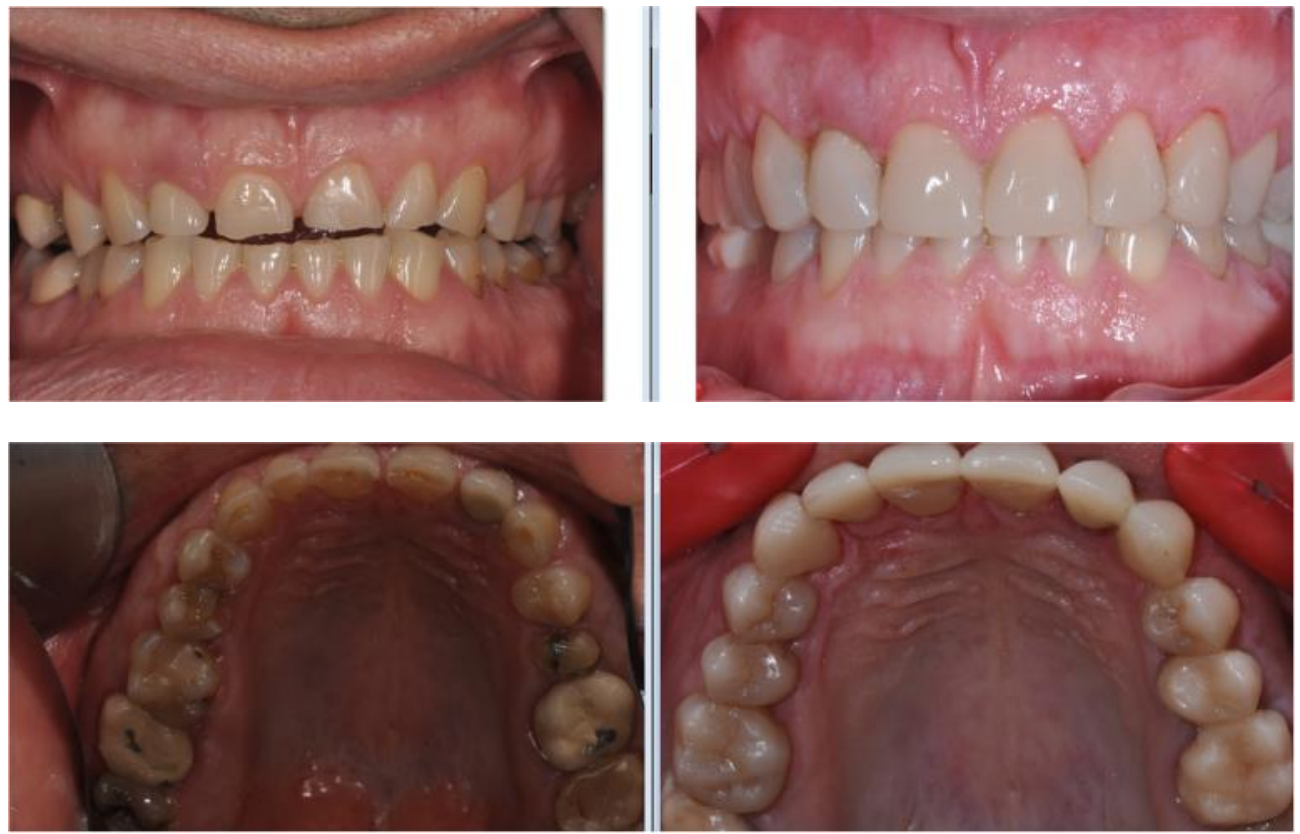

Figures 17 and 18 Comparison between before and after situations

\section{Discussion}

The presented treatment sequence up to the stage of finalized provisionals resolved several major problems related to full-mouth rehabilitation with a VDO increase. With the new centric position at the vertical dimension determined according to the rest position and the phonetic test together with the new 
anterior crown length and appearance, the complexity of the treatment is reduced to appropriate preparation, impression taking and fixation technique. Many dilemmas and possible mistakes are left behind without using any irreversible preparation of dental tissues, by following a simple protocol and use of a small amount of transparent silicon and a syringe of composite.

The treatment of advanced tooth wear is with no doubt one of the most difficult ones in prosthetic and restorative dentistry. Moreover, all epidemiological studies indicate that the need for this kind of treatments will dramatically increase in the near future. In order to perform this treatment, the clinician has to resolve a number of dilemmas: what type of restorations to make, how much to increase the DVO, how to determine the new centric position, how long should the new anterior teeth be, how to obtain the best function and aesthetic effect, will the new occlusal and gingival line require additional preprosthetic procedures, etc.

The use of the modified Le Gall jig allows the clinician since the very first visit to have a clear vision regarding the:

- horizontal plane and model orientation

- midline

- $\quad$ centric position of the lower jaw validated using several methods

- $\quad$ length of the central incisors

- $\quad$ maximum possible increase of the VDO

- $\quad$ tooth display during rest and during speaking.

All provisionals should be fabricated in order to "fit in" the established parameters and controlled using shimstock. The foil should pass neither between the newly fabricated provisional antagonistic couple, nor between the jig and its lower antagonists.

\section{Centric position}

The choice of the new centric position of the mandible (Le Gall preferred not to call it centric relation for many reasons) is a crucial step when increasing the VDO. The advantage of the present protocol is that it allows the validation of the chosen position using several different methods long known from total prosthetics. Thanks to the use of articulating paper and the jig even the tiniest discrepancies between the positions registered using different methods can be visualized since markings will not coincide.

How much can we raise the VDO

Another important issue is how much can we increase the VDO. From a laboratory standpoint the bigger increase in VDO will provide more space for the restorative material. The minimal VDO increase will be a function on the minimal occlusal clearance necessary for the mechanical stability of the restoration and will be material-dependent. For monolithic lithium disilicate this minimal occlusal thickness is $0,8-1,0$ for each tooth (9). If that clearance cannot be provided by VDO increase, an occlusal preparation should be performed. However, the patient must be able to close their lips with the new restorations and VDO and this is the absolute limit to the VDO increase (10). Some authors' protocols include recommendations for the VDO to be done primarily on an articulator. Our strong belief is that this important decision should not be delegated to the laboratory but instead must be taken in the clinic, taking into consideration phonetics, lips closure VDO, the rest position VDO, and the incisal display during speech with the strong cooperation and agreement of the patient.

\section{Patient comfort trial}

This paper demonstrates only the provisional phase of the treatment. The provisional restorations should be approved by the patient and validated for several weeks in the mouth in order to proceed with the finalization of the treatment. No consensus exists on how long the patient's comfort should be tested in 
order to validate the new VDO. According to the Bulgarian literature, the period is between two and three weeks (11), the Geneva team tested it for one month (12), other teams - for several months (13). Once the new VDO and centric position have been validated, the subsequent treatment is much more straightforward compared to the multiple dilemmas presented by the initial situation.

\section{Definitive rehabilitation choices}

Several choices exist regarding the type of restoration for the final phase of the treatment. The approach characterizing this type of treatments that was practiced only a decade ago, comprising root canal treatments and full crown preparation, is no longer justified (2). The so-called three-step technique is nowadays a quasi-universal approach to such cases. Much less invasive restoration types should be chosen. Such is for instance the "Minimally Invasive Prosthetic Procedure" (MIP) advocated by Fradeani (14) allowing full crown preparation within the thickness of enamel restored using adhesively fixed high strength all ceramic crowns. Another alternative restoration type is the vestibulo-occlusal overlays preferred by other authors (10), allowing for minimal or no-prep adhesively bonded restorations. These can be done with or without interproximal preparation. Little is known in terms of long term effects about which is a better solution - preparing or not interproximal surfaces when VO overlays are the restorations of choice. Since tissue preservation is the key with all the above-described new techniques and approaches, permanent restoration in the present clinical case will combine both approaches. Crowns will be chosen on teeth with preexisting crowns or previous root canal treatment, while VO overlays will be placed on vital and more preserved teeth following the tissue economy principle.

\section{Provisionals fixation choices}

Different approaches exist regarding the way of fixation of the provisional constructions. Interim crowns fixed with provisional cement are the best choice when the teeth are prepared for full crowns, however with less retentive restoration types (veneers or onlays) debonding of provisionals is more likely to occur. Spot etching might be used for short term fixation. However, if used for long-term treatment, marginal discoloration and sensitivity might occur due to microleakage. Gurel (15) describes a technique named "Aesthetic pre-evaluative temporaries" (APT) where provisionals made using a transparent silicon impression from the wax-up are bonded on the unprepared tooth surface and left for some period for aesthetic and functional validation by the patient. In the present clinical case we adopted this approach but extended the trial period to several months to make sure that the changes will not disturb the masticatory system proper functioning and comfort.

\section{Conclusion}

The increasing frequency of patients with severely worn dentition requires a feasible and reliable protocol for their treatment. On the other hand, a minimally invasive approach is a must in modern dentistry. Combining both requirements, the presented technique with modified Le Gall's jig offers a useful and timesparing treatment option manageable even by a general practitioner who would otherwise be intimidated by such complicated cases

\section{Acknowledgement}

We express our sincere gratitude to the team of DENTABLY Dental Lab and to the master dental technician Stefan Petrov for the sophisticated fabrication of the laboratory steps. 


\section{References}

1. Salas M.M., Nascimento G.G., Huysmans M.C., Demarco F.F.. Estimated prevalence of erosive tooth wear in permanent teeth of children and adolescents: an epidemiological systematic review and meta-regression analysis. J Dent. 2015 Jan; 43 (1): 42-50. doi: 10.1016/j.jdent.2014.10.012. Epub 2014 Nov 8.

2. Vailati F., Belser U.C. Full-Mouth Adhesive Rehabilitation of a Severely Eroded Dentition: The Three-Step Technique Part 2. The Europian Journal Of Esthetic Dentistry 2008; 3: 30-44.

3. Le Gall, M. G., J. F. Lauret. Occlusion et function, une approche clinique rationnelle (p.46). - CdP Editions, 2002.

4. Jaeggi T., Lussi A. Prevalence, incidence and distribution of erosion; Monogr Oral Sci. 2014; 25: 55-73.

5. Van Roekel N.B. Gastroesophageal reflux disease, tooth erosion, and prosthodontic rehabilitation: A clinical report; J Prostodont 2003; 12: 255-259

6. G rel G., Yerusalmi B. M., Shayder A. Monolithic CAD/CAD Porcelain Laminate Veneers with External Staining QDT 2013 book.

7. Le Gall M, Joerger R, Bonnet B. Où et comment situer l'occlusion ? Relation centrée ou position de déglutition guidée par la langue ? Cah Prothèse 2010; 150: 33-46.

8. Fradeani M. Barducci G. Esthetic rehabilitation in fixed prosthodontics. Volume 2. Prosthetic treatment: a systematic approach to esthetic, biologic, and functional integration; Quintessence Publishing. 2008: p.70.

9. Bacherini L., Brennan M., Bocabella L., Vigiani P. Esthetic Rehabilitation of a Severely Discolored Dentition with Minimally Invasive Prosthetic Procedures (MIPP) QDT 2013 book.

10. Lasserre J-F. Comprendre l'augmentation de DVO dans les approaches minimales invsives des traitements de l'usure et des anomalies de l'émail. Alpha Omega news France, No138.

11. Peev TP. Diagnostics and treatment of occlusal dental wear. Doctoral thesis, Faculty of Dental Medicine- Sofia, 1993.

12. Vailati F., Belser U.C. Full-Mouth Adhesive Rehabilitation of a Severely Eroded Dentition: The Three-Step Technique. Part 2 The European Journal Of Esthetic Dentistry 2008; 3, 2: 128-146.

13. Schweiger J. Edelhoff D. Noninvasive Provisional Restorations Using High-Density Polymers QDT 2013 book.

14. Fradeani M, Bacherini L, Brennan M. Esthetic rehabilitation of a severely worn dentition with minimally invasive prosthetic procedures (MIPP). Int J Periodontics Restorative Dent 2012; 32: 135-147. 
15. G rel G. Predictable and precise tooth preparation techniques for porcelain laminate veneers in complex cases International Dentistry SA 9, 1: 30-40.

\section{Corresponding author:}

Assistant Professor Ivan Chakalov; Prosthetic Dentistry, Faculty of Dental Medicine, Medical University - Sofia; Georgi Sofiyski blvd., 1431 Sofia, Bulgaria;

Telephone number: +359888937264

E-mail address: uanio@yahoo.com 